\title{
Implementation of an Educational Program and an Antibiotic Order Form to Optimize Quality of Antimicrobial Drug Use in a Department of Internal Medicine
}

\author{
I. C. Gyssens ${ }^{1,2 *}$, W. L. Blok ${ }^{1}$, P. J. van den Broek ${ }^{3}$, Y. A. Hekster ${ }^{4}$, \\ J. W. M. van der Meer ${ }^{1}$
}

\begin{abstract}
In a study designed to evaluate the effects of an educational program and an antibiotic order form on the quality of antimicrobial drug use, a prospective analysis was conducted in the department of internal medicine of a 948-bed university hospital. Following a quality-of-use review of all consecutive courses of antimicrobial drugs prescribed during four weeks, an educational program was conducted and an antibiotic order form introduced. After four years, an identical review was performed. In the first review, 109 (31\%) of 347 patients were prescribed antimicrobial drugs. Only $40 \%$ of the prescriptions were considered definitely appropriate, and $13 \%$ were considered unjustified. There was a certain degree of underutilization, and only $67 \%$ of clinical isolates were susceptible to empirical therapy. In the review performed after intervention, 164 (21\%) of 796 patients were given antimicrobial drugs. Defined daily doses per 100 bed days increased from 59.8 to 72.6 . Fifty-three percent of the prescriptions were judged optimal, and only $9 \%$ were judged unjustified. Ninety percent of the clinical isolates were susceptible to empirical therapy. After one year, compliance with the antibiotic order forms on a voluntary basis reached $77 \%$, documenting $86 \%$ of antimicrobial drug costs. As a result, the antibiotic order form will be useful for surveillance, if logistic support is provided by the pharmacy. The combination of several measures leads to improved quality of use. As correctly predicted by the first evaluation, improvement in quality resulted in increased drug consumption by fewer patients and a higher cost per bed day.
\end{abstract}

The increasing cost of antimicrobial drug consumption, the reports of inappropriate use of antimicrobial drugs $(1,2)$, and the worldwide increase in resistance (3) are the main factors on which the antibiotic policy measures of the $1990 \mathrm{~s}$ are based. Recently, national antimicrobial drug consumption data from European hospitals were published by Janknegt et al. (4). Dutch university hospitals had a rather low consumption of antimi-

\footnotetext{
${ }^{1}$ Department of General Internal Medicine, and ${ }^{4}$ Department of Clinical Pharmacy, University Hospital Nijmegen, PO Box 9101, 6500 HB Nijmegen, the Netherlands.

2 Department of Medical Microbiology and Infectious Diseases, University Hospital Rotterdam, Dr. Molewaterplein 40, 3015 GD Rotterdam, the Netherlands.

${ }^{3}$ Department of Infectious Diseases, University Hospital
} Leiden, PO Box 9600, 2300 RC Leiden, the Netherlands. crobial drugs (44.3-46.6 defined daily doses per 100 bed days) compared with German and Belgian hospitals, which seems to be reflected by lower resistance rates in the Netherlands. However, little is known about the quality of antimicrobial drug use. Criteria for evaluation of the quality of antimicrobial drug use are well established $(5,6)$. Many strategies to improve prescribing have been described. Education as the sole intervention strategy to improve quality has not always been successful (7). Antibiotic order forms filled in by prescribers have been used to monitor use and to influence prescribing habits in the USA $(8,9)$, but experience in Europe is very limited or unpublished.

We studied the use of antimicrobial drugs in the department of internal medicine of a large univer- 
sity hospital. The aims of the study were to define the patterns of antimicrobial drug use in terms of quality and costs; to evaluate the effect of an educational program; and to measure the value of an antibiotic order form.

\section{Patients and Methods}

Setting. The University Hospital Nijmegen is a 948-bed teaching hospital. The study took place in selected units of the Department of Internal Medicine, involving 114 of the 183 beds. Some of these units were highly specialized, such as the unit of nephrology, where organ transplants are performed. A large unit of general internal medicine had an older patient population. Over the study years, the number of beds remained unchanged. The number of kidney transplantations increased from 90 in the first study year to 111 in the last. At the time of the first review, the hospital formulary listed 20 parenteral and 26 oral antibacterial drugs. In that year, antimicrobial drugs accounted for $22 \%$ of the total drug budget of 14 million guilders (Dfl; approximately US $\$ 7$ million). Hospital formulary drugs were kept in ward-based stocks. Pharmacy technicians supplied the drugs to the wards on a twice-weekly basis. Nonformulary drugs had to be ordered from the pharmacy on special order forms for individual patients. Computerized consumption figures were available for different wards, but not for individual patients.

Antimicrobial Utilization Review. The first prospective review, performed over a four-week period, started in October 1989. After a period of intervention, a second review was conducted over a six-week period in 1993. The units I (general internal medicine) and $\mathrm{N}$ (nephrology) were selected to measure the effect of an educational program and an antibiotic order form, while the unit of pulmonary diseases $(P)$, where no order form had been used, was studied as a control.

Prospective quality-of-use studies were performed by an infectious diseases physician and junior clinical pharmacists, who visited the wards daily and collected data from medication charts on all patients receiving antimicrobial drugs. This method has also been applied to surgical departments (10). Clinical information was retrieved from patients' records. Abstracts were made of each consecutive antimicrobial drug course. A course was defined as an episode of clinical or suspected infection or increased risk of infection in which prescription(s), either consecutively or in combination, were written to treat or prevent this particular infection. Infections were defined according to the criteria of the Centers for Disease Control (11). Nosocomial infection was defined as active infection that was not present or incubating at the time of admission. Antimicrobial drug use was analyzed quantitatively in defined daily doses (DDDs). The DDD represents the average therapeutical dose for an adult for the standard indication (12). Costs were calculated in guilders using a method of global drug-cost calculation, which includes costs of administration and monitoring (13). Results of microbiological tests were obtained directly from the laboratory.

A quality evaluation of individual prescriptions was performed by two independent experts in infectious diseases, termed reviewer 1 and 2 . They placed prescriptions into categories using well-defined criteria. The most authoritative classification is that developed by Kunin et al. (5) in 1973. We adapted their criteria in order to be able to evaluate each parameter of importance associated with antimicrobial drug use. Several subcategories have been added to the original criteria (6). Briefly, prescriptions can be judged definitely appropriate (category I) or unjustified (category V), or the records can be insufficient for categorization (category VI). Other prescriptions are placed in categories II, III, and IV, indicating inappropriate use. Inappropriate prescriptions can be allocated to several categories at the same time: incorrect dose (IIa), incorrect interval (IIb), incorrect route (IIc), duration too long (IIIa), or duration too short (IIIb). If relevant, the reviewers cited an alternative agent deemed better due to higher efficacy (IVa), lower toxicity (category IVb), lower cost (IVc), or narrower spectrum (IVd). Overall costs of actual and alternative policies were compared to project savings that would result from changes in policy. Because only one expert, reviewer 1, was involved in the education and policy changes of the intervention, the detailed evaluation results of only reviewer 1 are presented.

Apart from the above-named criteria used by reviewers for evaluation, two additional parameters known to be associated with good antimicrobial use, i. e., mention of the suspected microorganism in the medical record and correct monitoring of potentially toxic antimicrobial drugs, were evaluated separately. We also determined whether the microorganism isolated was susceptible to the drug prescribed initially as empirical treatment and if streamlining (14) was performed after microbiology results became available.

Intervention strategies. The principal goal of the interventions was to improve the quality of use. An educational program was conducted, which consisted of weekly teaching sessions of $1 \mathrm{~h}$ at which one or two cases were presented by an infectious diseases specialist. The sessions were attended by $10-25$ medical students, residents, and junior and senior staff members of the department of internal medicine. The case presentations were interactive and focused on diagnosis and antimicrobial therapy. Resistance patterns, virulence, and principles of pharmacokinetics (dosing, frequency) were discussed. Furthermore, during that period, two infectious diseases specialists were available at the weekly grand rounds for clinical consultation. The order form was introduced during these teaching sessions. Newsletters reported to prescribers their actual rate of compliance with the order forms, and prescribers could compare their compliance data with those of colleagues from other wards (data were presented anonymously).

An update of the antimicrobial drug formulary containing guidelines for prophylaxis and therapy was issued. In addition to this educational program, an antibiotic order form was introduced in units I and N in 1992. The form was introduced on a voluntary basis, and details are described elsewhere (15). In brief, physicians were asked to categorize prescriptions as prophylaxis, empiric therapy, or directed therapy. They had to state the (presumed) site of infection, (presumed) causative microorganism, planned duration of the course, and parameters such as weight, serum creatinine, and presence of allergy. A limited number of formulary antimicrobial drugs and dosage regimens were printed on the form and could be ticked off. The order forms were gathered for surveillance purposes only; the information on the forms was not used for intervention.

Statistical Analyses. Generally, chi-square tests were applied to establish systematic differences. Agreement between the reviewers was assessed by $\kappa$ coefficients. 
Table 1: Demographic data and quantitative antimicrobial drug (AD) use in three units before (first review) and after (second review) intervention.

\begin{tabular}{|c|c|c|c|c|c|c|c|c|c|c|c|c|c|c|c|}
\hline & \multicolumn{8}{|c|}{ First reviewa } & \multicolumn{7}{|c|}{ Second reviewb } \\
\hline & \multicolumn{2}{|c|}{ Unit 1} & \multicolumn{2}{|c|}{ Unit N } & \multicolumn{2}{|c|}{ Unit $P$} & \multicolumn{2}{|c|}{ Total } & Unit I & \multicolumn{2}{|c|}{ Unit $N$} & \multicolumn{2}{|c|}{ Unit $P$} & \multicolumn{2}{|c|}{ Total } \\
\hline Total no. of patients & 253 & & 52 & & 42 & & 347 & & 576 & 138 & & 82 & & 796 & \\
\hline No. of patients with $A D(\%)$ & 57 & (23) & 30 & (58) & 22 & (52) & 109 & (31) & $85 \quad(15)$ & 45 & (33) & 341 & $(41)$ & 164 & (21) \\
\hline No. of bed days & 2200 & & 430 & & 379 & & 3009 & & 2372 & 626 & & 326 & & 3324 & \\
\hline Total no. of $A D$ courses & 73 & & 39 & & 23 & & 135 & & 98 & 72 & & 38 & & 208 & \\
\hline Prophylactic courses (\%) & 6 & (8) & 2 & (5) & 0 & (0) & 8 & (6) & $4 \quad(4)$ & 27 & (38) & 4 & (11) & 35 & (17) \\
\hline Therapeutic courses (\%) & 67 & (92) & 37 & (95) & 23 & $(100)$ & 127 & (94) & $94 \quad(96)$ & 45 & (62) & 34 & (89) & 173 & (83) \\
\hline Courses in combination (\%) & 19 & (26) & 8 & $(21)$ & 5 & (22) & 32 & (24) & $15 \quad(15)$ & 7 & (10) & 4 & (11) & 26 & (13) \\
\hline Total consumption (no. of DDD) & 1231 & & 252 & & 315 & & 1798 & & 1394 & 672 & & 413 & & 2479 & \\
\hline $\mathrm{DDD} / 100$ bed days & 56 & & 58.6 & & 83.1 & & 59.8 & & 58.8 & 107.3 & & 126.7 & & 74.6 & \\
\hline $\mathrm{DDD} /$ course & 16.9 & & 6.5 & & 13.7 & & 13.3 & & 14.2 & 9.3 & & 10.9 & & 11.9 & \\
\hline Total cost (Dfl) & 18,966 & & 10,874 & & 9773 & & 39,613 & & 35,720 & 17,338 & & 13,219 & & 6,277 & \\
\hline Total cost/bed day (Dfl) & 8.6 & & 25.3 & & 25.8 & & 13.2 & & 15.1 & 27.7 & & 40.6 & & 19.9 & \\
\hline
\end{tabular}

a One-month review.

b Six-week review.

DDD, defined daily doses; Dfl, Dutch guilders; I, general internal medicine; N, nephrology; P, pulmonary medicine.

\section{Results}

First Review. A detailed analysis of the data from the units of general internal medicine (I), nephrology $(\mathrm{N})$, and pulmonary diseases $(\mathrm{P})$ is presented in Table 1 to allow comparison with the data of the second review. In units I and P,24\% of the patients were older than 70 years. Thirty-three percent of the patients had concomitant corticosteroid use, and $<10 \%$ were receiving concomitant immunosuppressive drugs. In unit $\mathrm{N}$, none of the patients was older than 70 years, and $72 \%$ of the patients had concomitant corticosteroid therapy with immunosuppressive drugs.

In the first review, 109 (31\%) of 347 patients were administered antimicrobial drugs (Table 1). There was a large difference in consumption between unit $\mathrm{I}$ and both units $\mathrm{N}$ and $\mathrm{P}$, where more than half of the patients were treated with antimicrobial drugs. The patients in unit $\mathrm{N}$ had the highest consumption in terms of courses $/ 100$ bed days, but due to renal function impairment, most patients had dose reductions that resulted in a relatively low consumption, expressed in DDD/100 bed days (Table 1 ). Unit $\mathbf{P}$ had the highest consumption in DDD/100 bed days, and more than three-quarters of the drugs were administered orally; in the other units, the majority of the drugs were administered parenterally. Combinations of two or more drugs were prescribed in $24 \%$ of all courses.

The courses were almost exclusively categorized as therapy (Table 1). The types of infections treated with antimicrobial drugs are presented in Table 2. Respiratory tract infections were the most frequent type of infection treated with anti-

Table 2: Types of infections treated with therapeutic courses of antimicrobial drugs in three units before and after intervention.

\begin{tabular}{|c|c|c|c|c|c|c|c|c|}
\hline \multirow[t]{3}{*}{ Site of infection } & \multicolumn{8}{|c|}{ Percent } \\
\hline & \multicolumn{4}{|c|}{ First review ${ }^{a}$} & \multicolumn{4}{|c|}{ Second review ${ }^{b}$} \\
\hline & $\begin{array}{l}\text { Unit I } \\
(n=67)\end{array}$ & $\begin{array}{l}\text { Unit N } \\
(n=37)\end{array}$ & $\begin{array}{l}\text { Unit P } \\
(n=23)\end{array}$ & $\begin{array}{l}\text { Total } \\
(n=127)\end{array}$ & $\begin{array}{l}\text { Unit I } \\
(n=94)\end{array}$ & $\begin{array}{l}\text { Unit N } \\
(\mathrm{n}=45)\end{array}$ & $\begin{array}{l}\text { Unit P } \\
(n=34)\end{array}$ & $\begin{array}{l}\text { Total } \\
(n=173)\end{array}$ \\
\hline Respiratory tract & 30 & 32 & 78 & 39 & 24 & 22 & 82 & 35 \\
\hline Urinary tract & 9 & 32 & 0 & 14 & 9 & 29 & 6 & 13 \\
\hline Fever of unknown origin & 1 & 0 & 4 & 2 & 4 & 0 & 0 & 2 \\
\hline Blood & 21 & 8 & 0 & 13 & 13 & 13 & 3 & 11 \\
\hline Abdomen & 12 & 3 & 0 & 7 & 17 & 0 & 0 & 9 \\
\hline Skin/soft tissue & 9 & 5 & 4 & 7 & 14 & 7 & 0 & 9 \\
\hline Miscellaneous/unknown & 18 & 19 & 13 & 17 & 19 & 29 & 9 & 20 \\
\hline
\end{tabular}



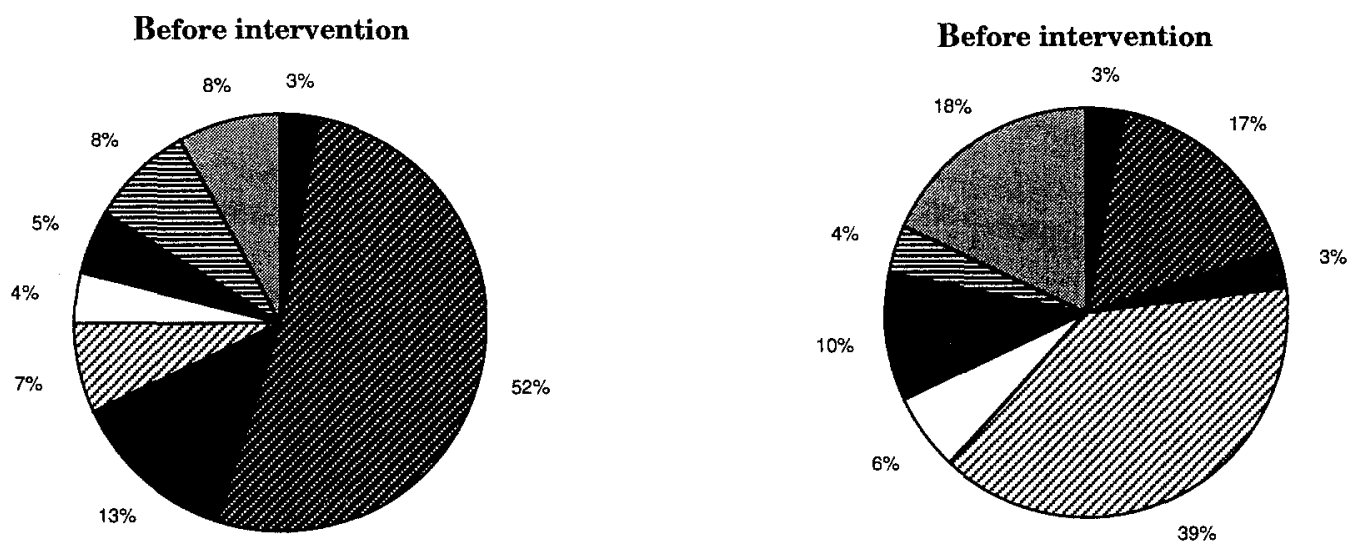

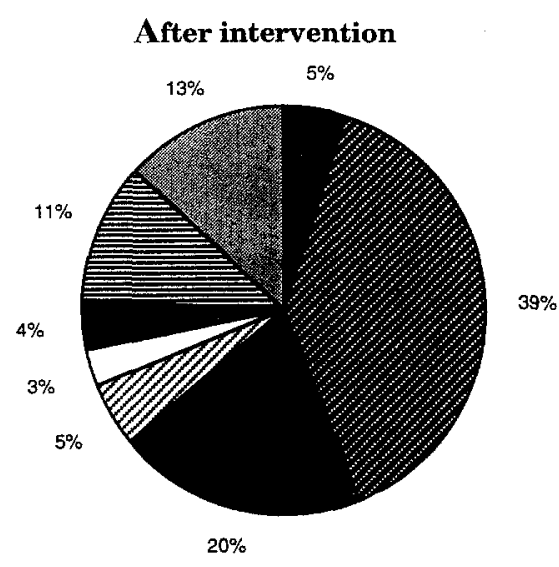

\% total DDD

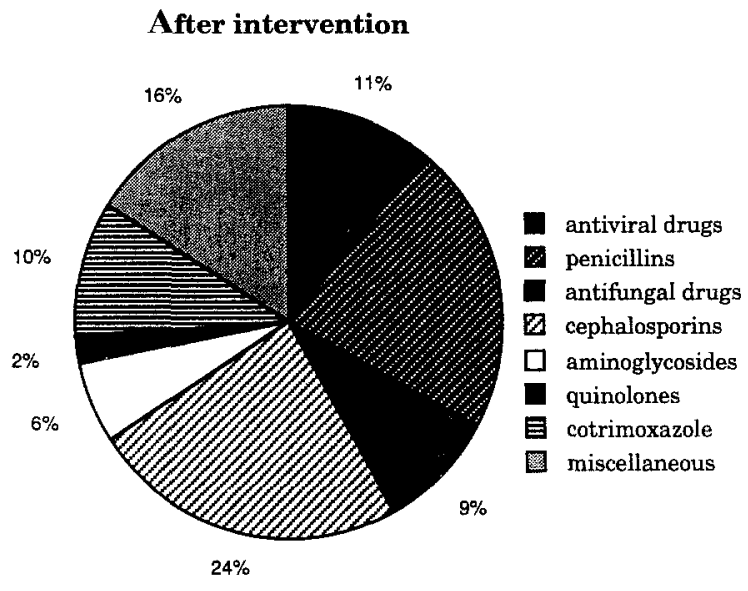

\% total cost

Figure 1: Distribution of antimicrobial drug consumption before and after intervention. Left, distribution in percent total defined daily doses (DDD); right, distribution in percent total cost.

microbial drugs $(39 \%)$. Thirty-four percent of these infections were nosocomial. In unit $\mathrm{N}$, urinary tract infections were most frequent $(32 \%)$. Thirty-three percent of all infections treated could be classified as nosocomial. The consumption of therapeutic antimicrobial drugs, expressed as percent total DDD, is presented in Figure 1. Penicillins accounted for half of the overall consumption. Ninety-seven percent of penicillins consisted of older penicillins (penicillin G, amoxicillin). Twenty-three percent of cephalosporins were of the first generation (mainly cefazolin and cephalexin), $45 \%$ of the second generation (mainly cefuroxime), and $32 \%$ third generation (exclusively ceftazidime). Antifungal and antiviral drugs accounted for $16 \%$.

One course cost an average of Dfl 293. Cost figures are presented in Table 1. Overall cost/bed day. was Dfl 13.2. However, there were large differenc- es in the cost/bed day. Although unit $\mathrm{N}$ used expensive drugs, its cost/bed day and the cost/ course were relatively low due to the previously mentioned dose modifications for impaired renal function. The distribution of antimicrobial drug costs for units I, N, and P combined is given in Figure 1. More than half of the costs were due to consumption of cephalosporins and penicillins.

The most frequently isolated microorganisms were Escherichia coli and Klebsiella spp. together $(20 \%)$, Staphylococcus aureus $(6 \%)$, and Staphylococcus epidermidis $(6 \%)$. In Figure 2, the relationship between antimicrobial drug prescribing and microbiology laboratory utilization is illustrated. Microbiology laboratory testing could be studied in 123 of 127 therapeutic courses for which the site of infection was known. In three-quarters of these courses, microbiology tests were performed. The tests yielded a relevant microorganism in 
Microbiology laboratory utilization

before/after intervention

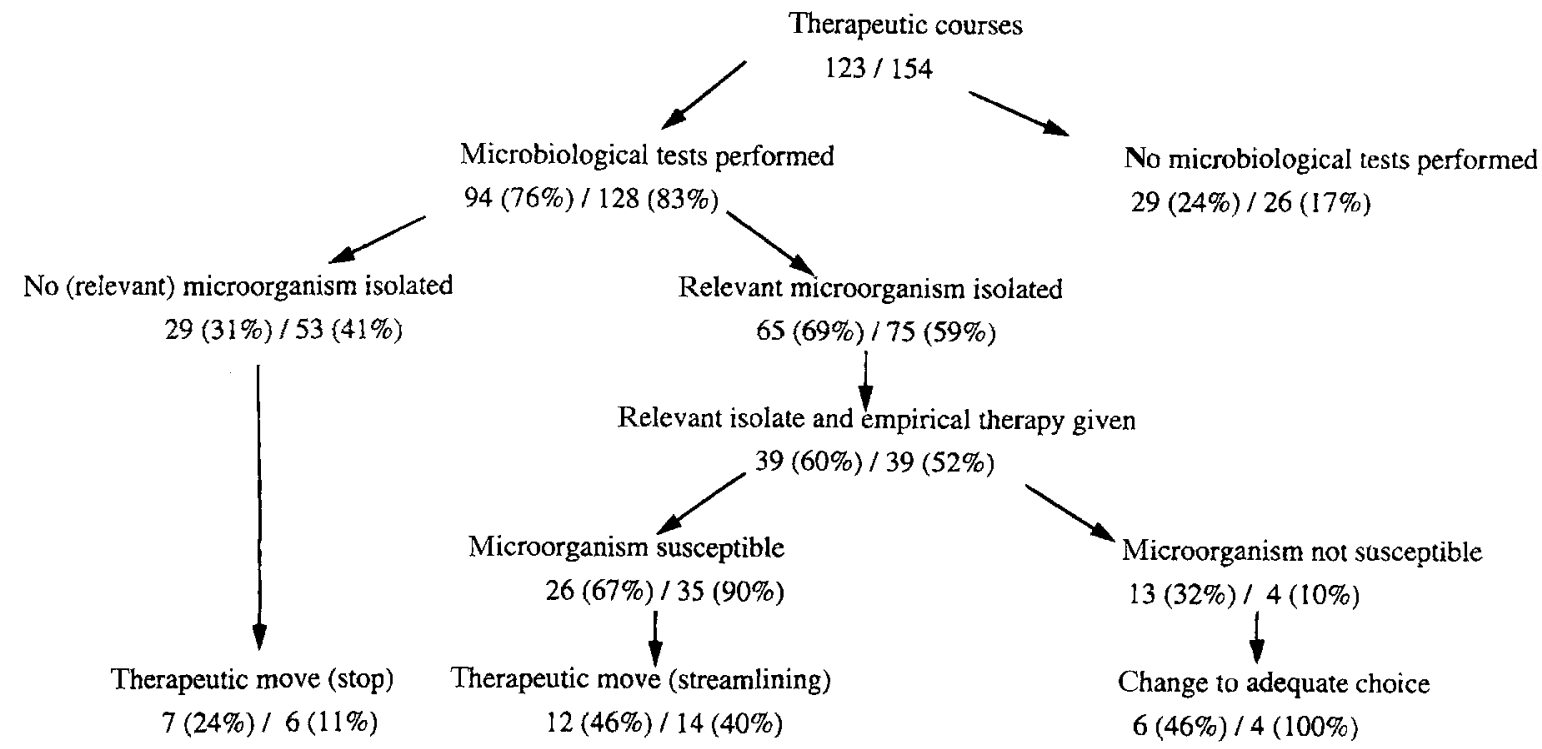

Figure 2: Microbiology laboratory utilization and the impact of the laboratory results on prescribing. Only therapeutic antimicrobial drug courses were studied ( $n=123$ before intervention, $n=154$ after intervention).

$69 \%$. Only $67 \%$ of these microorganisms were susceptible to the drugs prescribed as empiric treatment. In fewer than half of the cases where the microorganism was not susceptible, therapy was changed to an adequate spectrum. Streamlining of empirical therapy was done in $46 \%$ of the cases. In only $10 \%$ of those who were eligible for streamlining would it have been possible.

Figure 3 summarizes detailed categories of evaluation of all prescriptions by the two reviewers. Two hundred fifty-nine prescriptions could be evaluated (categories II, III, and IV could be assigned simultaneously to a prescription). There was only moderate agreement (ignoring category VI) between the reviewers $(\kappa=0.40)$. Agreement was higher when only categories I (definitely appropriate) and $V$ (unjustified) were considered $(\kappa=0.56)$. Reviewer 2 judged more prescriptions unjustified than reviewer 1 in all three units. He also judged fewer prescriptions definitely appropriate. Table 3 shows the results of the evaluation by reviewer 1 for the three units. Fewer than half of the prescriptions were judged definitely appropriate (category I), and $13 \%$ were judged unjustified (category V). More than one-third of prescriptions could be optimized (category II-IV).

Cost projections were made. Elimination of prescriptions judged as unjustified by reviewer 1 would result in a savings of only $8 \%$. The low frequency of less costly alternatives (category IVc; $5 \%$ ) or alternatives with a narrower spectrum (category IVd; $3 \%$ ) did predict minor savings. Moreover, because duration of therapy was almost never considered too long (category IIIa) and was even judged too short (category IIIb) in $2 \%$ of prescriptions, no savings were expected by an improvement in prescribing. Finally, the combination of category IIa (incorrect dose, most often too low; $15 \%$ ), category IVa (more effective alternative wanted; $19 \%$ ), and the fact that only $67 \%$ of the microorganisms were susceptible to drugs started empirically suggested the need for higher doses of drugs with a broader spectrum. It was anticipated that implementing the policy of reviewer 1 would result in a cost increase.

In $16(23 \%)$ of 70 empirical courses, the suspected microorganism was mentioned in the medical record. Unit P scored best, with six (38\%) of 16 . Formulary drugs were used in $86 \%$ of cases $(88 \%$ in unit $\mathrm{I}, 76 \%$ in unit $\mathrm{N}, 93 \%$ in unit $\mathrm{P}$ ). In all 12 courses of gentamicin lasting $>72 \mathrm{~h}$, serum concentrations were measured. Courses were given as thrice-daily regimens. In four of 12 courses, a peak concentration of $<5 \mathrm{mg} / \mathrm{l}$ was measured (considered too low), and in four of 12, a trough concentration of $>1 \mathrm{mg} / \mathrm{l}$ was measured (consid- 

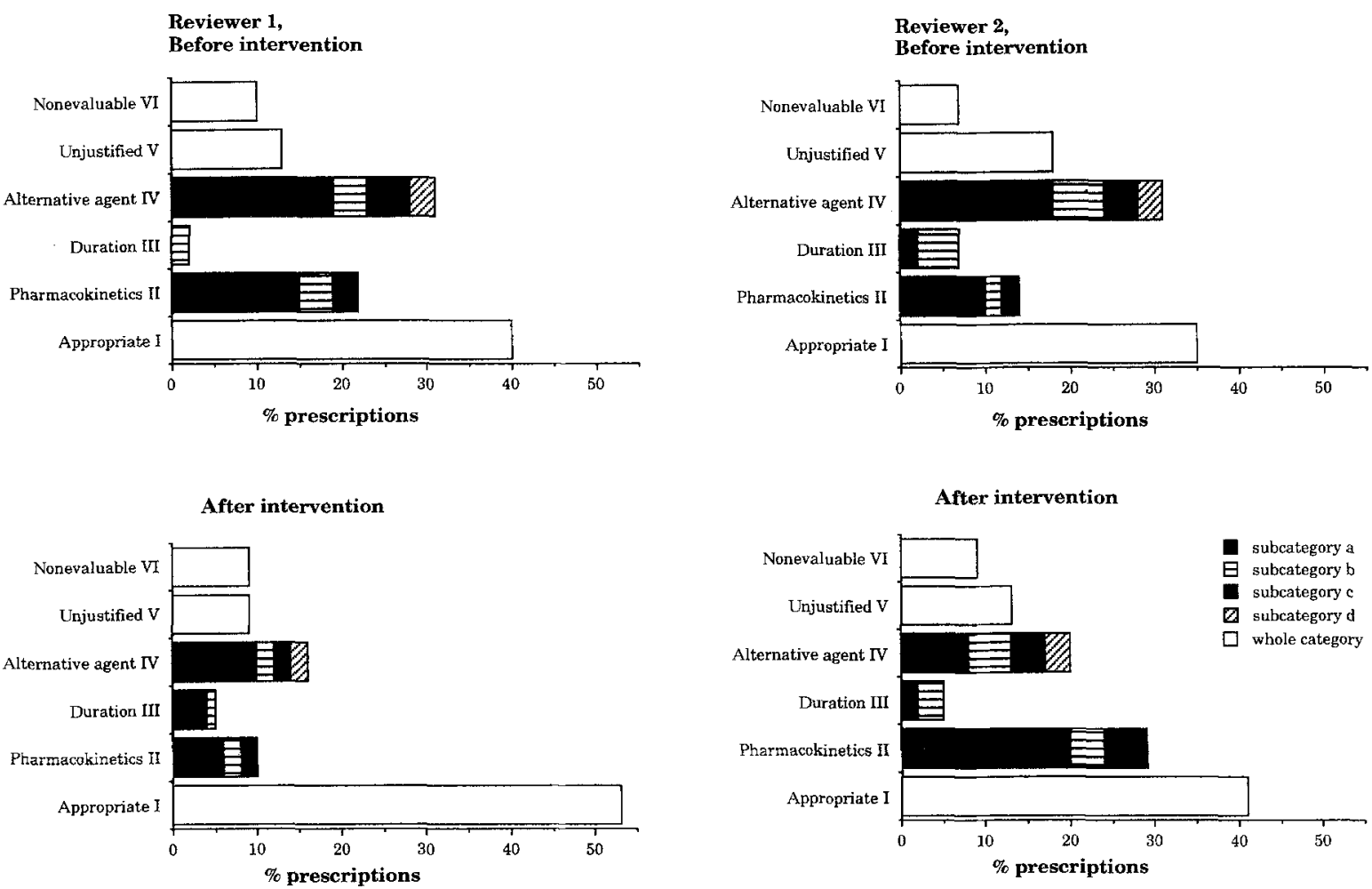

Figure 3: Quality evaluation of antimicrobial drug prescriptions by two reviewers before $(n=259)$ and after $(n=332)$ intervention. Left, reviewer 1; right, reviewer 2. Categories of evaluation: I, definitely appropriate; lla, incorrect dose; Ilb, incorrect dosage frequency; Ilc, incorrect route, IIla, too long; III b, too short; IVa, alternative agent more effective; IVb, alternative agent less toxic; IVc, alternative agent less expensive; IVd, alternative agent less broad spectrum; V, unjustified.

ered too high). In all courses the dose and/or frequency was modified. In three courses the second peak concentration was still $<5 \mathrm{mg} / \mathrm{l}$.

Antimicrobial Drug Surveillance between the Two Reviews. Between the first review and the second review, an update of the antimicrobial drug formulary was issued, and five antimicrobial drugs were added to the formulary: amoxicillin clavulanate, ciprofloxacin, trimethoprim, fluconazole, and itraconazole. Five older drugs were removed.

Surveillance data of the pharmacy showed that expenses for antimicrobial drugs remained stable in 1990 but had increased by $35 \%$ in 1991 and by $45 \%$ in 1992 in units I and N. In those units, the average length of stay had decreased by one day between 1989 and 1993.

Table 3: Evaluation by reviewer 1 of the quality of antimicrobial drug use in three units before and after intervention.

\begin{tabular}{|c|c|c|c|c|c|c|c|c|}
\hline \multirow[t]{3}{*}{ Evaluation } & \multicolumn{8}{|c|}{ Percent } \\
\hline & \multicolumn{4}{|c|}{ First review ${ }^{\mathrm{a}}$} & \multicolumn{3}{|c|}{ Second review ${ }^{b}$} & \multirow[b]{2}{*}{$\begin{array}{l}\text { Total } \\
(\mathrm{n}=332)\end{array}$} \\
\hline & $\begin{array}{l}\text { Unit I } \\
(n=143)\end{array}$ & $\begin{array}{l}\text { Unit N } \\
(n=71)\end{array}$ & $\begin{array}{l}\text { Unit P } \\
(n=45)\end{array}$ & $\begin{array}{l}\text { Total } \\
(n=259)\end{array}$ & $\begin{array}{l}\text { Unit I } \\
(n=162)\end{array}$ & $\begin{array}{l}\text { Unit N } \\
(n=121)\end{array}$ & $\begin{array}{l}\text { Unit P } \\
(n=49)\end{array}$ & \\
\hline $\begin{array}{l}\text { Definitely appropriate } \\
\text { (category I) }\end{array}$ & 41 & 30 & 56 & 40 & 59 & 47 & 47 & 53 \\
\hline $\begin{array}{l}\text { Unjustified (category V) } \\
\text { Inappropriate }\end{array}$ & 11 & 13 & 18 & 13 & 12 & 2 & 18 & 9 \\
\hline (category II-III-IV) & 35 & 48 & 24 & 37 & 23 & 37 & 24 & 29 \\
\hline Unevaluable (category VI) & 13 & 10 & 2 & 10 & 6 & 14 & 10 & 9 \\
\hline
\end{tabular}

a One-month review.

b Six-week review.

I, general internal medicine; $N$, nephrology; $P$, pulmonary medicine. 
The purchase cost of most antimicrobial drugs decreased between the times of the two reviews. The cost of cephalosporins decreased by $10 \%$, and the cost of ciprofloxacin and vancomycin by $25 \%$. The antifungal drugs fluconazole and itraconazole, used on a compassionate-use basis in the first review, became part of the hospital formulary, resulting in a cost increase for antifungal drugs. To analyze the consequences of these complex cost changes, a second in-depth review was done.

Second Review. Age distribution and concomitant use of corticosteroid and immunosuppressive drugs were similar in the second review.

Table 1 shows the comparison of quantitative data. Comparing the units I, N, and P, there was a reduction in the overall proportion of patients receiving antimicrobial drugs, from 31 to $21 \%$ (Table 1). The difference was significant in unit I $(\mathrm{p}=0.006)$ and unit $\mathrm{N}(\mathrm{p}=0.002)$, but not in unit $P(p=0.25)$. Overall consumption increased in terms of $\mathrm{DDD} / 100$ bed days. Prescription of combinations of two or more antimicrobial drugs decreased from 24 to $13 \%$ in the three units.

Six percent of the courses were intended for prophylaxis in the first review and $17 \%$ after intervention (Table 1). This consumption increased mainly because the Department of Nephrology started post-transplantation prophylaxis against Pneumocystis carinii with cotrimoxazole.

Respiratory tract infections were the most frequent type treated with antibiotics (Table 2), and the sites of infection were very similar. Twentythree percent of respiratory tract infections were nosocomial. The consumption of penicillins, cephalosporins, and aminoglycosides remained stable (Figure 1). The increase in consumption was caused by a $90 \%$ increase in the use of antiviral and antifungal drugs and to slight increases in the use of a variety of agents. The consumption of the newly added formulary drugs amoxicillin/clavulanate and ciprofloxacin did not increase.

A comparison of cost parameters before and after intervention is shown in Table 1. The total cost/bed day increased from Dfl 13.2 to 19.9. An increase in cost/bed day occurred in all three units, but was most pronounced in units I and P. A higher cost/DDD, representing the use of more expensive drugs, and a higher cost/course were noted in unit I only. A comparison of the distribution of antimicrobial agent costs before and after intervention is shown in Figure 1. The increase in prophylaxis with oral cotrimoxazole had only a minor in- fluence on the costs, as it represented only $4 \%$ of the total cost.

During the second review, the data on the antibiotic order forms of units I and $\mathrm{N}$ were compared with the data collected using the in-depth method. Defining compliance as the total number of antibiotic order forms collected/total number of prescriptions, compliance was $77 \%$ in unit I and $50 \%$ in unit N. In $98(58 \%)$ of 170 courses, at least one order form had been completed. At least one form was filled in for $39 \%$ of prophylactic courses and for $61 \%$ of therapeutic courses. In unit I, $86 \%$ of total antimicrobial drug costs were documented by order forms, but in unit $\mathrm{N}$, it amounted to only $41 \%$.

Site of infection and categorization as prophylaxis or therapy were well documented in $98 \%$ of cases in unit I and in $90 \%$ in unit N. In empirically started therapy, a suspected agent was mentioned in $70 \%$ and $62 \%$ of cases in these respective units. However, some items were regularly omitted. History of allergy was most frequently left blank ( $44 \%$ ), followed by weight $(41 \%)$ and creatinine level (31\%). Only $33 \%$ of forms were filled in completely. In $98 \%$ of the forms, the formulary drugs preprinted on the form were chosen. On $80 \%$ of the forms in unit I, preprinted doses and dosage intervals were ticked off. In unit $\mathrm{N}$, only $33 \%$ of the preprinted regimens were used. This was probably due to dose and/or dosing interval adaptations for impaired renal function, although this reason was only mentioned on half of those forms.

After the intervention, $96 \%$ of the antimicrobial drug prescriptions were formulary drugs in unit I and $\mathrm{N}$; in unit $\mathrm{P}$, it amounted to $89 \%$. The utilization of microbiological tests in therapeutic courses increased from 76 to $83 \%$ in the second review (Figure 2). The yield of relevant microorganisms was lower in the second review (59\% vs. $69 \%$ ). After intervention, $90 \%$ of the isolates were susceptible to empirical therapy, compared with $67 \%$ before intervention. All empirical therapy was changed to directed therapy with an adequate spectrum after culture results were known.

Three hundred thirty-two prescriptions could be evaluated. Figure 3 allows comparison of detailed categories of evaluation of all prescriptions by the two reviewers before and after intervention. After intervention, agreement (ignoring category VI) between the reviewers was also partial $(\kappa=$ $0.27)$. An antimicrobial drug prescription could be classified as inappropriate because alternative 
regimens were available that were considered a better choice by one of the reviewers. For example, streamlining cefazolin to flucloxacillin for the specific therapy of staphylococcal infection was considered optimal therapy by one reviewer because of its narrow antistaphylococcal spectrum. The other reviewer agreed with the continuation of the empirically prescribed cefazolin, which is also effective, economical, and has fewer side effects such as infusion-related phlebitis. In this evaluation, reviewer 2 also considered more prescriptions unjustified than reviewer 1 . He judged fewer prescriptions definitely appropriate; this was true for all units.

Comparison of the quality evaluation for the three units by reviewer 1 before and after the intervention is shown in Table 3. According to reviewer 1 , the overall proportion of prescriptions that were considered definitely appropriate (category I) increased from 40 to $53 \%$. Unjustified prescriptions (category V) decreased from 13 to $9 \%$. There were relatively fewer prescriptions classified in categories II to IV (inappropriate). The main parameters of quality that improved were the choice of a more effective antimicrobial drug (category IVa), optimal dosage (category IIa), and dosage frequency (category IIb). The differences were statistically significant for the total prescriptions $(\mathrm{p}=0.01)$. There were also significant differences in quality before and after the intervention in unit $\mathrm{I}(\mathrm{p}=0.003)$ and unit $\mathrm{N}(\mathrm{p}=$ $0.002)$, but not in unit $P(p=0.91)$, where no order form was used. According to reviewer 2, there were significant differences in quality before and after the intervention in unit $\mathrm{N}$ only (data not shown).

\section{Discussion}

Improper antimicrobial drug use contributes to emergence of resistant strains and to waste of health resources. Guidelines for improving the use of antimicrobial agents in hospitals issued by the Infectious Diseases Society of America have recommended various tactics (16), including formation of antimicrobial agent teams to select drugs and organize educational programs. The recommendations further include implementation of methods that improve selection, such as the use of antibiotic order forms, automatic stop orders, limited susceptibility reporting, concurrent monitoring of use and specific audits. Finally, regulation of the promotional efforts by the pharmaceutical industry is recommended.
In our hospital, as in almost every other hospital in the Netherlands, a limited hospital formulary of antimicrobial drugs composed by antimicrobial agent teams and limited susceptibility reports by the laboratory have been functional for many years. In the first audit described in this study, we could not detect any major misuse cited by Marr et al. (16), such as excessive duration of therapy or concurrent use of more than two drugs. In fact, we found a certain degree of underuse. We projected that implementing a policy to improve quality would result in a cost increase. Although quantitative use after the intervention increased in terms of DDD/100 bed days, the proportion of patients who were receiving antimicrobial drugs was lower than before. This finding can be explained by the use of higher dosages and/or longer duration of treatment restricted to patients with proven infections and by the shorter length of stay. Total cost/bed day increased for the same reason and because of the justified use of more expensive drugs with a broader spectrum.

Apart from correctly estimating the cost of future policy changes, the method of quality evaluation by two independent reviewers showed that there can be different alternatives for an antimicrobial therapy regimen. Part of the disagreement between reviewers can be explained not only by the preference of different alternatives, but also by reviewer 1's involvement in the education program. Personal factors (reviewer 2 was more strict in his evaluation of all units) may also have played a role.

Physicians continue to use antimicrobial drugs inappropriately because knowledge of the basic principles of good antimicrobial therapy is lacking. However, education as the single intervention strategy to improve quality has not always been successful (7). The order form can help in the proper selection of the antimicrobial drug regimen in terms of choice, dose, and frequency. Another advantage of the form is that it requires the prescriber to state the suspected causative microorganism. Without an order form, it is not clear whether the prescriber does not know the potentially causative agent or merely fails to mention it in the record, unless the prescriber is interviewed (17).

The order form was successful in stimulating the use of standardized dosing frequencies, an effect described by others (9). In this way, the order form may have contributed to improved quality of prescribing. Because we used a combination of intervention strategies (education, update of the formulary, antibiotic order forms), it is not possible to es- 
timate the effect of each intervention separately. The goal of the order form was to facilitate surveillance. This was aided by having the prescriber provide the data for drug utilization, as the indepth reviews had been very time-consuming. No immediate action was undertaken to improve prescribing at the time of the collection of the forms (15). The order form was generally well accepted by prescribers. Voluntary compliance was high in unit I, with a coverage of more than $80 \%$ of antimicrobial drug costs. Better compliance with the form can be achieved by making the form mandatory and controlled by the pharmacy (18). In such a setting, the order form offers more opportunities. It can be used to assign automatic discontinuation dates (19), or to initiate an infectious diseases consultation for restricted drugs (20). Computer system links between orders of antimicrobial drugs and data of the microbiology laboratory can identify patients whose antibiotic therapy is inappropriate in relation to sensitivity data (21). With the support of the hospital pharmacy, the form could be a useful tool for antimicrobial drug surveillance and for intervention in European hospitals in the future.

\section{Acknowledgements}

The pharmacists-in-training and the pharmacy technicians of the Department of Clinical Pharmacy are gratefully acknowledged for their help with the collection of the data. We thank R. de Graaf (Department of Medical Statistics, University of Nijmegen) for his help with the analysis of the data

\section{References}

1. Kunin $C M$, Staehr Johansen $K$, Worning AM, Daschner FD: Report of a symposium on use and abuse of antibiotics worldwide. Reviews of Infectious Diseases 1990, 12: $12-19$.

2. Dunagan WC, Woodward RS, Medoff G, Gray JL III, Casabar E, Lawrenz CA, Spitznagel E, Smith MD: Antibiotic misuse in two clinical situations: positive blood culture and administration of aminoglycosides. Reviews of Infectious Diseases 1991, 13: 405-412.

3. Cohen ML: Epidemiology of drug resistance: implications for a post-antimicrobial era. Science 1992, 257: 1050-1055.

4. Janknegt R, Wijnands WJA, Caprasse $M$, Brandenburg W, Schuitenmaker MG, Stobberingh E: Antimicrobial drug use in hospitals in the Netherlands, Germany, and Belgium. European Journal of Clinical Microbiology \& Infectious Diseases 1993, 12: 832-838.

5. Kunin CM: Use of antibiotics: a brief exposition of the problem and some tentative solutions. Annals of Internal Medicine 1973, 79: 555-560.
6. Gyssens IC, Van den Broek PJ, Kullberg BJ, Hekster YA, Van der Meer JWM: Optimizing antimicrobial therapy. A method for antimicrobial drug evaluation. Journal of Antimicrobial Chemotherapy 1992, 30: 724-727.

7. Jones SR, Barks J: The effect of an educational program upon hospital antibiotic use. American Journal of the Medical Sciences 1977, 273: 79-85.

8. Avorn J, Soumerai SB, Taylor W, Wessels MR, Janousek $J$, Weiner M: Reduction of incorrect antibiotic dosing through a structured educational order form. Archives of Internal Medicine 1988, 148: 1720-1724.

9. Lipsy RJ, Smith GH, Maloney ME: Design, implementation, and use of a new antimicrobial order form: a descriptive report. Annals of Pharmacotherapy 1993, 27: 856-861.

10. Gyssens IC, Geerligs IEJ, Dony JMJ, Van der Vliet JA, Van Kampen A, Van den Broek PJ, Hekster YA, Van der Meer JWM: Optimising antimicrobial drug use in surgery: an intervention study in a Dutch university hospital. Journal of Antimicrobial Chemotherapy 1996, 38: 1001-1012.

11. Garner JS, Jarvis WR, Emori TG, Horan TC, Hughes JM: CDC definitions for nosocomial infections, 1988. American Journal of Infection Control 1988, 16: 128-140.

12. World Health Organisation: Anatomical therapeutic chemical (ATC) classification index, including defined daily doses (DDDs) for plain substances. WHO Collaborating Centre for Drug Statistics Methodology, Oslo, January 1993.

13. Gyssens IC, Lennards CA, Hekster YA, Van der Meer JWM: The cost of antimicrobial chemotherapy: a method for cost evaluation. Pharmaceutisch Weekbiad Science 1991, 13: 248-253.

14. Quintiliani R, Nightingale $\mathrm{CH}$, Crowe $\mathrm{HM}$, Cooper BW, Bartlett RC, Gousse G: Strategic antibiotic decisionmaking at the formulary level. Reviews of Infectious Diseases 1991, 13, Supplement 9: 770-777.

15. Blok WL, Gyssens IC, Hekster YA, Koopmans PP, Van der Meer JWM: Feasibility of an antibiotic order form. First experience in the department of internal medicine in a university hospital. Pharmacy World \& Science 1996, 18: 137-141.

16. Marr JJ, Moffet HL, Kunin CM: Guidelines for improving the use of antimicrobial agents in hospitals: a statement by the Infectious Disease Society of America. Journal of Infectious Diseases 1988, 157: 869-876.

17. Moss F, McNicol MW, McSwiggan DA, Miller DL: Survey of antibiotic prescribing in a district general hospital I. Pattern of use. Lancet 1981, ii: 349-352.

18. Echols RM, Kowalsky SF: The use of an antibiotic order form for antibiotic utilization review: influence on physicians' prescribing patterns. Journal of Infectious Diseases 1984, 150: 803-807.

19. Durbin WAJ, Lapidas B, Goldmann DA: Improved antibiotic usage following introduction of a novel prescription system. Journal of the American Medical Association 1981, 246: 1796-1800.

20. Scher KS, Bernstein JM, Arenstein GL, Sorensen C: Reducing the cost of surgical prophylaxis. American Surgeon 1990, 56: 32-35.

21. Pestotnik SL, Evans RS, Burke JP, Gardner RM, Classen DC: Therapeutic antibiotic monitoring: surveillance using a computerized expert system. American Journal of Medicine 1990, 88: 43-48. 